\title{
Effects of Excision of a Mass Lesion of the Precentral Region of the Left Hemisphere on Disturbances of Graphomotor Output
}

\author{
Oliver Tucha ${ }^{1}$, Lara Tucha ${ }^{1}$, Christian Smely ${ }^{2}$, Klaus W. Lange ${ }^{3}$ \\ ${ }^{1}$ Department of Clinical and Developmental Neuropsychology, University of Groningen, Groningen, The Netherlands; ${ }^{2}$ Department \\ of Neurosurgery, Lahr General Hospital, Lahr, Germany; ${ }^{3}$ Department of Experimental Psychology, University of Regensburg, Re- \\ gensburg, Germany. \\ Email: o.m.tucha@rug.nl
}

Received February $13^{\text {th }}$, 2012; revised March $15^{\text {th }}$, 2012; accepted April $17^{\text {th }}, 2012$

\begin{abstract}
In the present study, the effect of neurosurgery on graphomotor output of a right-handed female patient with a mass lesion of the precentral region of the left frontal lobe was reported. For examination of handwriting movements a digitizing tablet was used. Preoperatively, the patient showed longer movement times than healthy subjects and patients with lesions of the left frontal lobe without involvement of the precentral region. Furthermore, the analysis of kinematic data revealed a severe dysfluency of her handwriting. Postoperatively, a significant improvement of writing time and fluency of handwriting was observed. Since the integrity of handwriting plays an important role in everyday functioning, disturbances of handwriting movements should be objectified and reassessed in follow-up assessment using new techniques such as digitizing tablets.
\end{abstract}

Keywords: Brain Tumour; Neurosurgery; Handwriting; Kinematics; Motor Function

\section{Introduction}

The concept of quality of life has become established as an important consideration in the treatment of patients with intracranial mass lesions. Since alterations of mental functioning are common in patients with such lesions [1-3], the impact of neurosurgery, in particular on cognitive functions, has been examined in recent research [4-6]. As well as cognitive and sensory disturbances, patients often complain of motor disturbances. These disturbances affect not only gross motor functioning but also the coordination of fine motor movements [7].

\section{Case Report}

A 56-year-old right-handed woman with no history of neurological or psychiatric disease was admitted to the Department of Neurosurgery for excision of a meningioma in the left frontal lobe. The first symptoms she had complained of were fatigue and severe headache. Before admission, the patient had worked as a secretary for a small company. Over the four months previous to admission, she had experienced increasing problems with handwriting. She complained of an altered style of writing, with reduced legibility and of fatigue during writing which impaired her performance at work. No further disturbances of motor functioning, cognition or emotion were reported.

No deficits were found on preoperative neurological examination. Neuroradiological examination using a 1.5 Tesla MRI system (MAGNETOM Vision; Siemens, Erlangen, Germany) in triplanar imaging with common sequences (T1, T2, contrast-enhanced T1) revealed a mass lesion situated in the precentral region of the left hemisphere with local compressive effect and almost no perifocal edema. Homogenous enhancement of the tumour matrix and surrounding dural enhancement in coronar view implicated a small meningioma of the left convexity as tumour lesion (Figure 1). Tumour histology was neuropathologically confirmed.

On preoperative neuropsychological examination, the patient was alert, cooperative and well orientated. Her intellectual functions were average [8,9]. While, according to published normative data, no disturbances of memory functions, attention, working memory, verbal fluency functions or either visuo-spatial or visuo-constructive abilities were observed [10-16], her reaction time in a simple computerized reaction time task was increased [17]. In a detailed examination of language functions, no deficits were found with regard to spontaneous speech, Token Test, reading, naming or comprehension of speech 


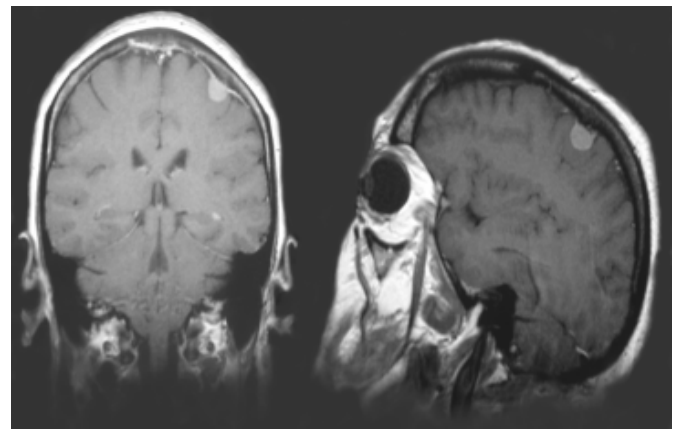

Figure 1. Left: Coronary view in contrast-enhanced T1weighted MRI; Right: Sagittal view in contrast-enhanced T1-weighted MRI.

[18]. Spelling was also unimpaired. However, her writing was laborious, effortful and slow.

\section{Methods}

\subsection{Apparatus and Measures}

For further examination of handwriting, a digitizing tablet (WACOM IV) with a specific pen containing a normal ink refill was used. Digitizing tablets make possible the examination of specific kinematic aspects of handwriting movements such as velocity and acceleration of single strokes. The analysis of velocity and acceleration of handwriting provides evidence of the existence of simple motor programs. It has been suggested that handwriting in healthy subjects is formed by the sequential activation of these motor programs which are probably stored in the form of a spatial code [19]. Single letterstrokes, the smallest relevant units of the writing process, are formed by open loop movements which are characterized by velocity profiles with only one peak (inversion of the direction) and a bell shaped course. Automated and non-automated handwriting movements can be distinguished from one another by the profiles of velocity and acceleration [20]. Only one inversion in velocity is expected when the writing movement is an open loop (fully automated or fluent). More than one inversion of velocity per stroke points to a disturbance of handwriting fluency or automation [21]. This means that the more inversions produced by subjects, the poorer they have mastered the movement.

The tablet used in the present examination had a maximum sampling rate of $200 \mathrm{~Hz}$. The position of the pen on the tablet, velocity and acceleration were measured continuously during writing. Data was stored on a personal computer connected to the tablet. Kinematic data were calculated and smoothed using nonparametric regression methods (kernel estimators) [22]. It was possible to localize the tip of the pen with an accuracy of 0.2 $\mathrm{mm}$ in both directions $(\mathrm{x} / \mathrm{y})$. Movements of the pen tip above the paper, up to a maximum of $1.3 \mathrm{~cm}$, could also be recorded. Data processing was performed with a computer program for the analysis of handwriting movements [23]. For examination, the patient was asked to write the sentence "Ein helles grelles Licht” (“A bright and glaring light") repeatedly. Before the start of these writing tasks, several practice trials were undertaken in order to familiarize the subjects with the writing tablet. The tablet was constructed to resemble a common desk pad in order that subjects could produce their usual handwriting. No restrictions of posture, speed or size of writing were imposed. The sentences were written on unlined white paper (size $297 \times 210 \mathrm{~mm}$ ). For data analysis, the total writing time (movement time) and the distance of the writing trace of the test sentence were recorded per trial. Movement time (in ms) was defined as the time between the first and final movement in the writing of the test sentence. Distance (in $\mathrm{mm}$ ) was defined as the distance covered by the pen during the writing of the test sentence. For further analysis, a mean movement time and a mean distance was calculated. Furthermore, the letter combination "ll" of the German words "helles" (bright) and "grelles" (glaring) were taken for the assessment of kinematic aspects of handwriting. The letter combination "ll" was chosen since these letters represent a simple letter combination which is usually written with the letters joined. Furthermore, while writing the letter combination "ll", the pen remains in contact with the tablet. In the evaluation of kinematic data, the mean number of inversions of the direction of the velocity (NIV) and acceleration profiles (NIA) of the letter combination "Il" were calculated. Kinematic analysis of the letter combination "ll" was performed, since the examination of the dynamic and static writing trace may often require its segmentation into meaningful units. From a motor viewpoint, single letters and in particular single strokes represent the smallest relevant units of the handwriting movement [19]. Data analysis focused on the vertical component of the strokes.

\subsection{Participants}

In order to exclude age-related impairments of handwriting movements, five right-handed female subjects aged 51 to 57 years without neurological or psychiatric diseases performed the same handwriting task. Furthermore, five right-handed female patients aged 54 to 58 years with histologically confirmed menigiomas of the left frontal lobe without involvement of the precentral region underwent the same procedure.

\section{Results}

With regard to movement distance, our patient (mean 
distance: $427 \mathrm{~mm}$ ) displayed no differences in comparison to healthy subjects (mean distance: 294 to $532 \mathrm{~mm}$ ) and patients with mass lesions of the left frontal lobe without involvement of the precentral region (mean distance: 385 to $452 \mathrm{~mm}$ ). However, she showed longer movement times (mean time: 14,983 ms) than both healthy subjects (mean time: 6170 to $8573 \mathrm{~ms}$ ) and the patient group (mean time: 6374 to $8727 \mathrm{~ms}$ ). In addition, the number of inversions of velocity (NIV) and acceleration profiles (NIA) were markedly increased in our patient (Figure 2). While healthy subjects (mean NIV: 4.0 to 4.4; mean NIA: 6.4 to 8.1) and patients with left frontal lesions without involvement of the precentral region (mean NIV: 4.0 to 4.8 ; mean NIA: 6.2 to 10.2 ) performed single letter-strokes by open loop movements, a severe dysfluency of handwriting, as reflected in a higher number of inversions in velocity and acceleration profiles, could be observed in our patient (mean NIV: 24.4; mean NIA: 39.9).

Four months after total surgical removal of the mass lesion the patient underwent a second examination using the same test procedures. Since drugs have been shown to affect fine motor movements such as handwriting movements [24-27], postoperative assessment was performed after the patient had completed courses of steroid and anticonvulsive medication. Neurological examination revealed no deficits. Postoperative neuroradiological examination showed a complete removal of the tumour mass. The patient complained of sporadic headache and disturbances of attention but mentioned that she had noticed an improvement in her handwriting. In comparison to the results of the preoperative assessment of cognitive functioning no significant alterations were found. While verbal fluency functions, language, memory (including working memory) and visuo-constructive abilities were undisturbed, the reaction time of the patient remained increased. However, kinematic analysis of handwriting revealed a significant improvement of writing time and fluency of handwriting. While, postoperatively, the distance of the writing trace of the test sentence was unchanged (mean distance: $442.8 \mathrm{~mm}$ ), the patient needed between 7466 and $8520 \mathrm{~ms}$ to complete the sentence. Furthermore, she displayed automated handwriting movements (mean NIV: 4.2) as indicated by a single inversion of the velocity profile per stroke (Figure 2). In addition, the number of inversions of acceleration profiles was markedly decreased (mean NIA: 10.4).

\section{Discussion}

The present results indicate that intracranial mass lesions of the precentral region of the left hemisphere may affect the automation of handwriting movements. This finding is not surprising in view of the anatomy of the precentral motor areas and its functions. The primary motor cortex, the supplementary motor cortex and the premotor cortex are involved in the processing of handwriting movements. In right-handed people, handwriting is controlled by the primary motor cortex of the left hemisphere. The supplementary motor cortex plays an important role in the programming and coordination of movement and posture. Although the functions of the premotor cortex are less well understood, there is some evidence that this cortical region controls the proximal movements that move the arm to targets. Therefore, more complex movement sequences, such as handwriting movements, can be executed under the control of the premotor cortex [28]. Furthermore, our findings are also consistent with the results of neuroimaging studies. It has been shown that automated handwriting movements of healthy right-handed subjects were related to an increased regional cerebral blood flow (rCBF) of the dorsal and ventral premotor cortex and the inferior and superior parietal lobule [29]. Yousry and colleagues [30] observed during non-automated handwriting movements in their right-handed subjects an additional activation in fMRI of the pre- and postcentral gyri of the right hemisphere. They also found additional activation in the precentral gyrus, middle frontal gyrus and middle occipital gyrus of the left hemisphere. Peinemann and colleagues [31] also reported a higher cortical activation (rCBF) of the left prefrontal cortex and the right anterior parietal lobule, including the postcentral gyrus, during non-automated handwriting. However, when their right-handed subjects were requested to perform automated handwriting movements, a higher activation of the left supplementary motor cortex and the hand area of the left primary sensorimotor cortex was observed.

We assume that the impairments of handwriting movements in our patient were the consequence of disturbed functioning of the motor system, including the primary motor cortex, the supplementary motor cortex and the premotor cortex. These areas were probably affected by increased intracranial pressure and compression of adjacent brain tissue caused by the mass lesion of the precentral region of the left hemisphere. As a result, the patient was unable to produce automated handwriting movements. She therefore attempted to compensate her deficits by producing highly controlled handwriting movements which are associated with a higher activation of the right pre- and postcentral gyri [30,31] and an impaired handwriting fluency [32,33]. Following surgical intervention, the detrimental effects of the space occupying lesion were ameliorated and fully automated handwriting movements were restored.

Handwriting in adults is a complex psychomotor ability 

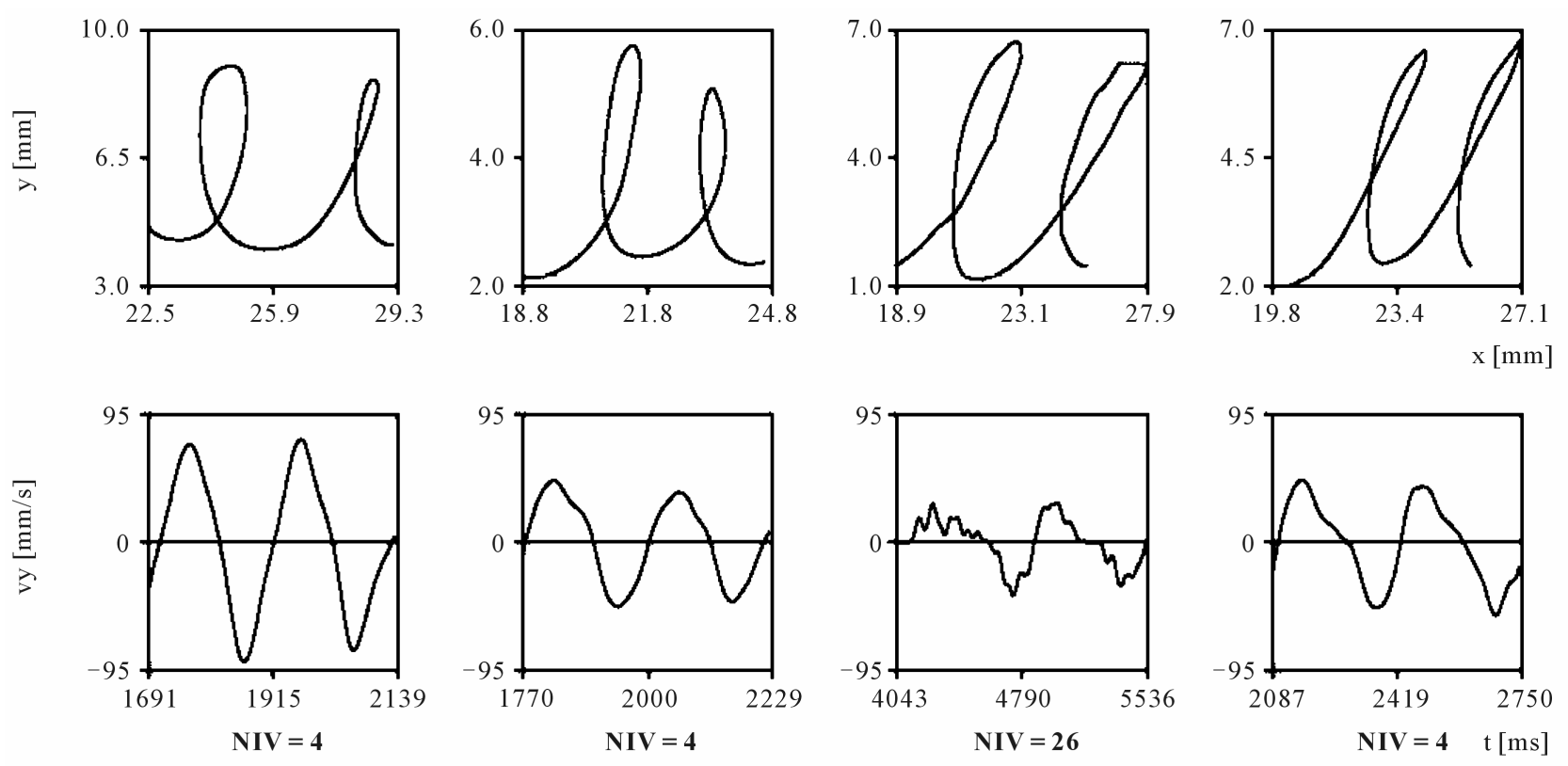

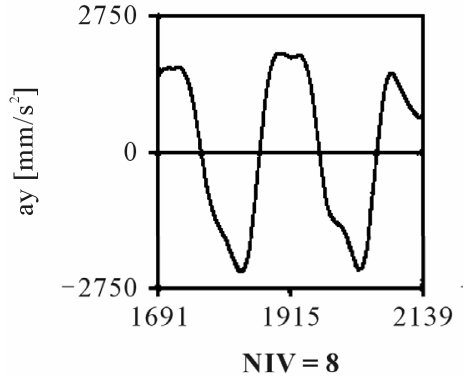

(a)

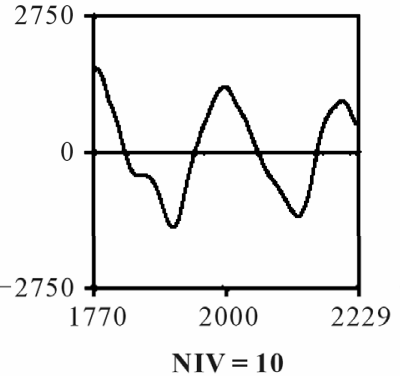

(b)

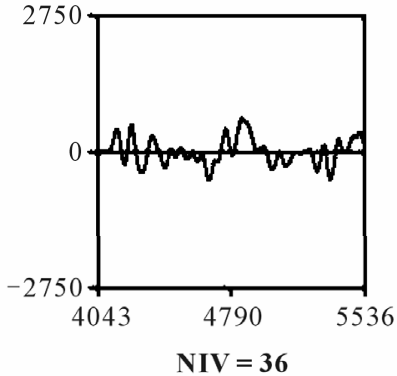

(c)

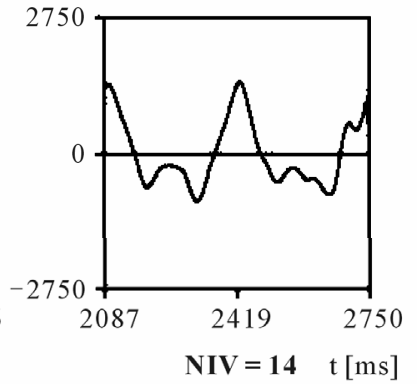

(d)

Figure 2. Left: Number of inversions in velocity (NIV) and acceleration (NIA) in a healthy participant (a) a patient with a left frontal lesion without involvement of the precentral region (b) and the patient with a left frontal lesion with involvement of the precentral region during preoperative (c) and postoperative assessment (d).

which constitutes a dynamic interplay of relatively slow horizontal movements of the lower arm, wrist movements and finger movements [34,35]. As well as semantic and syntactic demands, the process of handwriting necessitates the storage and retrieval of motor information, movement preparation, motor execution and the consideration of spatial requirements $[19,36]$. Therefore, both cognitive abilities and motor skills contribute to handwriting [37]. With regard to our patient, impairments of cognitive functioning can be ruled out since neuropsychological assessment using standardized test procedures revealed no disturbances in various aspects of cognition including memory, attention and both visuospatial and visuo-constructive functions.

Kinematic assessment of handwriting movements has also been shown to allow an objective analysis of psychomotor symptoms in patients with other clinical conditions including neurological or psychiatric diseases, such as Parkinson's disease, Huntington's disease, Alzheimer's disease, Attention Deficit Hyperactivity Disorder and Major Depression [25,33,38-41]. The clinical relevance of these kinematic assessments is supported by the finding that disturbances of handwriting may cause considerable handicap in everyday life and may even lead to loss of employment [42]. Since the integrity of handwriting plays an important role in everyday functioning, patients' complaints about disturbances of handwriting should be taken seriously. This is of particular importance in patients with intracranial mass lesions, since the mass lesions and their surgical treatment can cause all kinds of writing disturbances and abnormal writing behaviors such as agraphias or hypergraphia $[43,44]$. Furthermore, careful surgery involving intra-operative direct cortical stimulation (brain mapping) by using language and writing tasks demonstrated that language areas can be spared during tumor removal [45]. Since handwriting represents a motor act, a computerized registrations of handwriting movements which has been shown to pro- 
vide an objective, valid and reliable measure of psychomotor functioning [19,46-48] should also be part of the assessment of patients with handwriting disturbances. The assessment is easy to perform, is well tolerated by patients and takes only a few minutes. In summary, disturbances of drawing and handwriting movements of patients with space occupying lesions can be objectified using digitizing tablets. This technique could make an important contribution to the pre- and post-operative assessment of psychomotor functioning and an early referral of patients to motor rehabilitation programs.

\section{REFERENCES}

[1] M. J. B. Taphoorn, J. J. Heimans, F. J. Snoek, J. Lindeboom, B. Oosterink, J. G. Wolbers and A. B. M. F. Karim, "Assessment of Quality-of-Life in Patients Treated for Low-Grade Glioma-A Preliminary-Report," Journal of Neurology Neurosurgery and Psychiatry, Vol. 55, No. 5, 1992, pp. 372-376. doi:10.1136/jnnp.55.5.372

[2] O. Tucha, C. Smely and K. W. Lange, "Verbal and Figural Fluency in Patients with Mass Lesions of the Left or Right Frontal Lobes," Journal of Clinical and Experimental Neuropsychology, Vol. 21, No. 2, 1999, pp. 229-236. doi:10.1076/jcen.21.2.229.928

[3] O. Tucha, C. Smely, M. Preier and K. W. Lange, “Cognitive Deficits before Treatment among Patients with Brain Tumors," Neurosurgery, Vol. 47, No. 2, 2000, pp. 324333. doi:10.1097/00006123-200008000-00011

[4] M. J. B. Taphoorn and M. Klein, "Cognitive Deficits in Adult Patients with Brain Tumors," Lancet Neurology, Vol. 3, No. 3, 2004, pp. 159-168. doi:10.1016/S1474-4422(04)00680-5

[5] O. Tucha, C. Smely and K. W. Lange, "Effects of Surgery on Cognitive Functioning of Elderly Patients with Intracranial Meningioma," British Journal of Neurosurgery, Vol. 15, No. 2, 2001, pp. 184-188.

[6] Y. Yoshii, D. Tominaga, K. Sugimoto, Y. Tsuchida, A. Hyodo, H. Yonaha and S. Kushi, "Cognitive Function of Patients with Brain Tumor in Pre- and Postoperative Stage,” Surgical Neurology, Vol. 69, No. 1, 2008, pp. 5161. doi:10.1016/j.surneu.2007.07.064

[7] K. A. Jaeckle, M. E. Cohen and P. K. Duffner. "Clinical Presentation and Therapy of Nervous System Tumors," In: W. G. Bradley, R. B. Daroff, G. M. Fenichel and C. D. Marsden, Eds., Neurology in Clinical Practice, Butterworth-Heinemann, Oxford, 2000, pp. 1263-1279.

[8] G. Dahl, "Reduced Wechsler Intelligence Test," Hain, Meisenheim, 1972.

[9] D. Wechsler, "Wechsler Adult Intelligence Scale-Revised,” The Psychological Corporation, San Antonio, 1981.

[10] A. L. Benton, K. D. Hamsher and A. B. Sivan, "Multilingual Aphasia Examination,” AJA Associates, Iowa City, 1989.

[11] M. D. Lezak, D. B. Howieson and D. W. Loring, "Neuropsychological Assessment,” Oxford University Press, New
York, 2004

[12] M. N. Mitrushina, K. B. Boone and L. F. D’Elia, "Handbook of Normative Data for Neuropsychological Assessment,” Oxford University Press, New York, 1999.

[13] R. M. Reitan, "Validity of the Trailmaking Test as an Indication of Organic Brain Damage," Perceptual and Motor Skills, Vol. 8, 1958, pp. 271-276.

[14] A. Rey, "L'examen Psychologique Dans les cas D'encephalopathie Traumatique (Les Problems)," Archives de Psychologie, Vol. 28, 1941, pp. 215-285.

[15] P. A. Osterrieth, "Le Test de Copie d'une Figure Complexe,” Archives de Psychologie, Vol. 30, 1944, pp. 206356.

[16] D. Wechsler, "WMS-R: Wechsler Memory Scale-Revised,” The Psychological Corporation, San Antonio, 1987.

[17] P. Zimmermann and B. Fimm. "A Test Battery for Attentional Performance,” In: M. Leclercq and P. Zimmermann, Eds., Applied Neuropsychology of Attention: Theory, Diagnosis and Rehabilitation, Psychology Press, New York, 2002, pp. 110-151.

[18] W. Huber, K. Poeck, D. Weniger and K. Willmes, “Aachen Aphasia Test,” Hogrefe, Göttingen, 1983.

[19] A. J. W. M. Thomassen and G. P. Van Galen, "Handwriting as a Motor Task: Experimentation, Modelling, and Simulation,” In: J. J. Summers, Ed., Approaches to the Study of Motor Control and Learning, North-Holland, Amsterdam, 1992, pp. 113-144.

[20] O. Tucha, L. Tucha and K. W. Lange, "Graphonomics, Automaticity and Handwriting,” Literacy, Vol. 42, No. 3, 2008, pp. 145-155. doi:10.1111/j.1741-4369.2008.00494.X

[21] T. E. Eichhorn, T. Gasser, N. Mai, C. Marquardt, G. Arnold, J. Schwarz and W. H. Oertel, "Computational Analysis of Open Loop Handwriting Movements in Parkinson’s Disease: A Rapid Method to Detect Dopamimetic Effects," Movement Disorders, Vol. 11, No. 3, 1996, pp. 289-297. doi:10.1002/mds.870110313

[22] C. Marquardt and N. Mai, "A Computational-Procedure for Movement Analysis in Handwriting," Journal of Neuroscience Methods, Vol. 52, No. 1, 1994, pp. 39-45. doi:10.1016/0165-0270(94)90053-1

[23] N. Mai and C. Marquardt, "CS-Computer-Assisted Movement Analysis in Handwriting (Operational Manual)," MedCom, Munich, 1992.

[24] K. W. Lange, L. Mecklinger, S. Walitza, G. Becker, M. Gerlach, M. Naumann and O. Tucha, "Brain Dopamine and Kinematics of Graphomotor Functions," Human Movement Science, Vol. 25, No. 4-5, 2006, pp. 492-509. doi:10.1016/j.humov.2006.05.006

[25] O. Tucha, S. Aschenbrenner, P. Eichhammer, A. Putzhammer, H. Sartor, H. E. Klein and K. W. Lange, "The Impact of Tricyclic Antidepressants and Selective Serotonin Re-Uptake Inhibitors on Handwriting Movements of Patients with Depression,” Psychopharmacology, Vol. 159, No. 2, 2002, pp. 211-215. doi:10.1007/s002130100921

[26] O. Tucha and K. W. Lange, "Effects of Nicotine Chewing Gum on a Real-Life Motor Task: A Kinematic Analysis of 
Handwriting Movements in Smokers and Non-Smokers," Psychopharmacology, Vol. 173, No. 1-2, 2004, pp. 49-56. doi:10.1007/s00213-003-1690-9

[27] O. Tucha, S. Walitza, L. Mecklinger, D. Stasik, T. Sontag and K. W. Lange, "The Effect of Caffeine on Handwriting Movements in Skilled Writers," Human Movement Science, Vol. 25, No. 4-5, 2006, pp. 523-535. doi:10.1016/j.humov.2006.06.001

[28] C. Ghez, "Voluntary Movement,” In: E. R. Kandel, J. H. Schwartz and T. M. Jessel, Eds., Principles of Neural Science, Elsevier, New York, 1991, pp. 609-625.

[29] H. R. Siebner, C. Limmer, A. Peinemann, A. Drzezga, M. Schwaiger and B. Conrad, "Functional imaging of residual 'Left-Handedness' in Converted Left-Handers,” NeuroImage, Vol. 13, No. 6, 2001, p. S1253. doi:10.1016/S1053-8119(01)92567-4

[30] I. Yousry, M. Wiesmann, A. Nolte, T. Schenk, G. Fesl, T. A. Yousry and N. Mai, "Different fMRI Activation Patterns of Automated and Non-Automated Handwriting Movements,” NeuroImage, Vol. 9, No. 6, 1999, p. S497.

[31] A. Peinemann, P. Erhard, C. Baumgart, C. Mentschel, M. Schwaiger, B. Conrad and H. Siebner, "Fast Automatic Handwriting versus Show Controlled Handwriting: An Event-Related fMRI Study,” NeuroImage, Vol. 13, No. 6, 2001, p. S1236. doi:10.1016/S1053-8119(01)92550-9

[32] O. Tucha, S. Aschenbrenner and K. W. Lange, "Mirror Writing and Handedness," Brain and Language, Vol. 73, No. 3, 2000, pp. 432-441. doi:10.1006/brln.2000.2316

[33] O. Tucha, L. Mecklinger, S. Walitza and K. W. Lange, "Attention and Movement Execution during Handwriting,” Human Movement Science, Vol. 25, No. 4-5, 2006, pp. 536-552. doi:10.1016/j.humov.2006.06.002

[34] F. J. Maarse and A. J. W. M. Thomassen, "Produced and Perceived Writing Slant-Difference between up and down Strokes,” Acta Psychologica, Vol. 54, No. 1-3, 1983, pp. 131-147. doi:10.1016/0001-6918(83)90028-8

[35] A. J. W. M. Thomassen and H. L. Teulings, "Constancy in Stationary and Progressive Handwriting," Acta Psychologica, Vol. 54, No. 1-3, 1983, pp. 179-196. doi:10.1016/0001-6918(83)90032-X

[36] M. G. Longstaff and R. A. Heath, "Space-Time Invariance in Adult Handwriting,” Acta Psychologica, Vol. 97, No. 2, 1997, pp. 201-214. doi:10.1016/S0001-6918(97)00015-2

[37] G. P. Van Galen, A. J. W. M. Thomassen and A. M. Wing, "Handwriting: A Movement Theme," Human Movement Science, Vol. 10, No. 2-3, 1991, pp. 163-164. doi:10.1016/0167-9457(91)90002-F

[38] J. G. Phillips, J. L. Bradshaw, E. Chiu and J. A. Bradshaw, "Characteristics of Handwriting of Patients with Huntingtons-Disease,” Movement Disorders, Vol. 9, No. 5, 1994, pp. 521-530. doi:10.1002/mds.870090504
[39] M. J. Slavin, J. G. Phillips, J. L. Bradshaw, K. A. Hall and I. Presnell, "Consistency of Handwriting Movements in Dementia of the Alzheimer's Type: A Comparison with Huntington's and Parkinson's Diseases," Journal of the International Neuropsychological Society, Vol. 5, No. 1, 1999, pp. 20-25. doi:10.1017/S135561779951103X

[40] H. L. Teulings and G. E. Stelmach, "Control of Stroke Size, Peak Acceleration, and Stroke Duration in Parkinsonian Handwriting,” Human Movement Science, Vol. 10, No. 23, 1991, pp. 315-334. doi:10.1016/0167-9457(91)90010-U

[41] J. J. M. van Hoof, B. J. M. Jogems-Kosterman, B. G. C. Sabbe, F. G. Zitman and W. Hulstijn, "Differentiation of Cognitive and Motor Slowing in the Digit Symbol Test (DST): Differences between Depression and Schizophrenia,” Journal of Psychiatric Research, Vol. 32, No. 2, 1998, pp. 99-103. doi:10.1016/S0022-3956(98)00057-0

[42] N. Mai and C. Marquardt, “Treatment of Writer's Cramp: Kinematic Measures as an Assessment tool for Planning and Evaluating Training Procedures,” In: C. Faure, P. Keuss, G. Lorette and A. Vinter, Eds., Advances in Handwriting and Drawing: A Multidisciplinary Approach, Telecom, Paris, 1994, pp. 445-461.

[43] T. Imamura, A. Yamadori and K. Tsuburaya, "Hypergraphia Associated with a Brain-Tumor of the Right Cerebral Hemisphere," Journal of Neurology Neurosurgery and Psychiatry, Vol. 55, No. 1, 1992, pp. 25-27. doi:10.1136/jnnp.55.1.25

[44] P. Scarone, P. Gatignol, S. Guillaume, D. Denvil, L. Capelle and H. Duffau, “Agraphia after Awake Surgery for Brain Tumor: New Insights into the Anatomo-Functional Network of Writing," Surgical Neurology, Vol. 72, No. 3, 2009, pp. 223-241. doi:10.1016/j.surneu.2008.10.074

[45] V. Lubrano, F. E. Roux and J. F. Demonet, "WritingSpecific Sites in Frontal Areas: A Cortical Stimulation Study," Journal of Neurosurgery, Vol. 101, No. 5, 2004, pp. 787-798. doi:10.3171/jns.2004.101.5.0787

[46] W. Hulstijn. "Writing and Drawing in Neuropsychology: Studies on Psychomotor Slowness,” In: M. L. Simner, C. G. Leedham and A. J. W. M. Thomassen, Eds., Handwriting and Drawing Research: Basic and Applied Issues, IOS Press, Amsterdam, 1996, pp. 203-214.

[47] R. Mergl, P. Tigges, A. Schroter, H. J. Moller and U. Hegerl, "Digitized Analysis of Handwriting and Drawing Movements in Healthy Subjects: Methods, Results and Perspectives,” Journal of Neuroscience Methods, Vol. 90, No. 2, 1999, pp. 157-169. doi:10.1016/S0165-0270(99)00080-1

[48] H. L. H. M. Teulings and A. J. W. M. Thomassen, "Computer-Aided Analysis of Handwriting Movements," Visible Language, Vol. 13, No. 3, 1979, pp. 218-231. 